\title{
Disease and Diagnosis
}

\section{Effects of Cerium Oxide Nanoparticles on Biochemical Parameters and Histopathological Changes in Lead- Intoxicated Rats}

\author{
Mohammad Reza Hajinezhad ${ }^{1}$, Shaghayegh Hajian Shahri' ${ }^{* \circledast}$, Abbas Rahdar $^{2}{ }^{\circledR}$, Hojjat Zamanian $^{1}$ \\ ${ }^{1}$ Department of Basic Veterinary Science, Faculty of Veterinary Medicine, University of Zabol, Zabol, Iran. \\ ${ }^{2}$ Department of Physics, Faculty of Science, University of Zabol, Zabol, Iran.
}

\begin{abstract}
Background: The present study was conducted to investigate the potential protective effect of cerium oxide nanoparticles (CeNPs) against lead acetate-induced toxicity.

Materials and Methods: In this study, 30 adult male Wistar rats were randomly divided into three groups and treated simultaneously, except for the normal control, for 5 weeks with lead acetate in drinking water $(1000 \mathrm{mg} / \mathrm{L})$. Control rats and negative control rats received saline intraperitoneally. At the same time, the third group was treated with intraperitoneal injections of CeNPs at the dose of $0.5 \mathrm{mg} / \mathrm{kg} 1$ week before lead administration, and continued with its administration. Finally, serum was obtained by the conventional methods and rats were sacrificed to obtain liver, heart, testis, and kidney tissue for histopathological examinations.

Results: The lead-treated group showed significant increases in blood urea nitrogen (BUN), serum creatinine, serum aspartate aminotransferase (AST), serum alanine aminotransferase (ALT), and malondialdehyde $(\mathrm{MDA})$ levels $(P<0.01)$. Lead-intoxicated rats treated with CeNPs showed a significant decrease in serum lactate dehydrogenase $(\mathrm{LDH}), \mathrm{AST}$, and ALT levels compared to the untreated negative control group $(P<0.01)$. The histopathological examination of liver and kidney tissues showed signs of lead-induced injuries, necrotic hepatocytes, and glomerulosclerosis. The CeNPs-treated group showed noticeable reductions in histopathological signs of lead-induced injuries. Lipid peroxidation levels were also lower in CeNPs-treated rats than negative controls $(P<0.05)$.

Conclusion: The current experimental study proved the protective effects of CeNPs in rats exposed to chronic lead-induced toxicity; however, more experiments are required to evaluate the possible side effects and interactions.

Keywords: Lead acetate, Cerium oxide nanoparticles, Liver, Kidney, Toxicity
\end{abstract}

*Correspondence to

Shaghayegh Hajian Shahri, Department of Basic

Veterinary Science, Faculty of Veterinary Medicine,

University of Zabol, Zabol, Iran.

Tel: 05431232274;

Email: hajinezhad@uoz.

ac.ir

Received: October 17, 2020, Accepted: December 6, 2020, ePublished: December 30, 2020

\section{Introduction}

Lead is a dense chemical element that belongs to the heavy metals and is one of the most common materials in the earth's crust. Exposure to lead through contaminated air, food, water, dust, or cosmetic products, in the longterm period, can induce lead poisoning that is a type of metal poisoning (1). The central nervous system is the most susceptible organ to lead poisoning. Symptoms of lead-induced neurotoxicity are neuropathic pain, diarrhea, abdominal pain, arthritis, irritability, memory loss, and tingling in the face skin (2).

Chelation therapy is the most recommended treatment for lead poisoning. Chelation therapy is the removal of lead by using medications that can bind the lead. Several chemical compounds are recommended for lead chelation, including succimer, dimercaprol, edetate, and calcium disodium (3). Therapeutic interventions and treatment protocols depend on the age of the patient and serum lead concentration. Recent studies have shown that certain minerals like zinc, magnesium, and calcium can interfere with lead absorption from the gastrointestinal tract (4).

Cerium (IV) oxide or cerium dioxide - an oxide of the rare metal cerium - is a pale white-yellow powder (5). The antioxidant potential of Cerium oxide is mainly due to the free radical scavenging activity of this element, which reduces oxidative stress-induced damage (6). Cerium oxide nanoparticles (CeNPs) have diverse industrial applications in such fields as electronics, medical engineering, orthopedic devices, diagnostic kits, and

(C) 2020 The Author(s). This is an open access article distributed under the terms of the Creative Commons Attribution License (http:// creativecommons.org/licenses/by/4.0/), which permits unrestricted use, distribution, and reproduction in any medium, provided the original work is properly cited. 
cosmetics $(7,8)$. The unique biomedical applications of CeNPs make these materials among the most used NPs in industry and medicine. Therefore, many scientists are investigating the efficacy of these NPs as new drug delivery agents (9). CeNPs - due to the enhanced bioavailability and few toxicities - are used to prepare antioxidant, anticancer, and energizing formulations (10). Previous studies have shown the anti-inflammatory, antibacterial, and antioxidant properties of CeNPs (11). There are multiple reports regarding the interaction of NPs with lead acetate-induced nephrotoxicity and hepatotoxicity (12). In previous studies, CeNPs have shown hepatoprotective, anti-inflammatory, antilipogenic, antitomour, and neuroprotective effects (13). As far as the researchers investigated, there is little data regarding the protective effects of CeNPs against lead-induced toxicity. Therefore, the present study was conducted to examine these effects.

\section{Materials and Methods \\ Materials}

The biochemical parameters such as serum creatinine, serum blood urea nitrogen (BUN), and serum liver enzymes were determined by using the commercial diagnostic laboratory kits (Pars Azmoon, Tehran, Iran). All biochemical analyses were performed using the Selectra pro-M auto-analyzer (Vital Scientific, Netherlands, Span Neren).

\section{Animal Grouping}

To perform biological experiments, 30 adult male Wistar rats $(221 \pm 25 \mathrm{~g})$ were randomly divided into three equal groups. The animals were obtained from the animal breeding colonies of the laboratory animal house of the University of Zabol, Zabol, Iran. Before performing the experiments, rats were housed in the experimental room to get used to the experimental situation. Animals were kept at standard conditions: $23 \pm 1^{\circ} \mathrm{C} ; 12 / 12$ hours light/ dark cycle, humidity 50\%-70\%, and optimum ventilation 12 times per hour. Rats received lead acetate for 5 weeks in drinking water $(1000 \mathrm{mg} / \mathrm{L})$. Control rats and negative control rats received saline intraperitoneally. At the same time, the lead-treated group received CeNPs intraperitoneally at the dose of $0.5 \mathrm{mg} / \mathrm{kg}$ for seven days before administrating lead and during lead treatment. The dose of CeNPs was selected based on previous experiments (14).

Finally, serum was obtained from the retro-orbital sinus of the eye by using an EDTA-free micro-hematocrit tube. After blood sampling, the animals were euthanatized by intraperitoneal injection of sodium pentobarbital 150 $\mathrm{mg} / \mathrm{kg}$ (15) and were dissected to obtain different organ tissues for histopathological investigation. The liver sections were sampled from the caudate lobe of liver. Kidney samples were obtained from the left kidneys and heart samples were obtained from the left ventricles.

\section{Determination of the Lipid Peroxidation}

Measurement of serum malondialdehyde (MDA) levels was measured according to the spectrophotometric method presented by Ohkawa et al with some minor changes. This method is fundamentally based on the chemical reaction between MDA and thiobarbituric acid powder. After chemical reaction, the absorbance of final solution was determined by a spectrophotometer device and the final optical density results were expressed as $\mathrm{nmol} / \mathrm{mL}(16)$.

\section{Histopathological Examination}

To perform the histopathological investigations, animals were sacrificed to separate the abdominal and thoracic organs such as the liver, kidney, and testis. The specimens were sliced into two separate pieces. One piece was immediately frozen for further lipid-peroxidation investigation, and the other piece was preserved in $10 \%$ neutral buffer formalin. Then, the specimens were sent to the pathology laboratory for paraffin embedding and serial block making. The paraffin blocks were sliced with a microtome device. After paraffin embedding, the obtained liver, kidney, and testis sections were stained by the hematoxylin-eosin ( $\mathrm{H} \& \mathrm{E})$ method for further investigations under the light microscope (Olympus, Tokyo, Japan).

\section{Statistical Analysis}

The data of serum BUN, MDA, aspartate aminotransferase (AST), alanine aminotransferase (ALT), and creatinine were statistically analyzed using SPSS Statistics for Windows, version 18.0 (SPSS Inc., Chicago, Ill., USA) and one-way analysis of variance. Multiple comparisons were performed by Tukey's post hoc test at $5 \%$ confidence interval $(\mathrm{CI})(P<0.05)$.

\section{Results and Discussion \\ Biochemical Results}

The serum biochemical changes of lead-intoxicated rats and normal control rats are shown in Table 1.

Lead-intoxicated rats had elevated levels of serum AST $(P<0.01)$, serum ALT $(P<0.01)$, serum creatinine $(P<0.01)$, and serum BUN $(P<0.001)$ compared to the healthy control rats (Table 1$)$. The serum MDA level was also higher in the lead-intoxicated rats compared to the control rats $(P<0.001)$. Statistical analysis also showed elevated serum lactate dehydrogenase $(\mathrm{LDH})$ levels in lead-treated rats compared to the normal control rats $(P<0.01)$. The serum biochemical parameters of rats, which received the NPs, revealed a decrease in serum biochemical parameters and lipid peroxidation content compared to the lead-treated rats (Table 1). However, serum AST level, serum BUN, and serum ALT level showed no significant changes than lead-intoxicated rats $(P>0.05)$. The histopathological investigation of control group paraffin sections showed normal morphology 
Table 1. Serum Biochemical Indexes and Serum Lipid Peroxidation of Control and Lead-Treated Rats

\begin{tabular}{lccc}
\hline \multirow{2}{*}{ Item } & \multicolumn{3}{c}{ Treatment } \\
\cline { 2 - 4 } & Control & Lead- intoxicated rats & Selenium NPs + Lead \\
\hline $\begin{array}{l}\text { MDA (nmol/ } \\
\mathrm{ml})\end{array}$ & $50.3 \pm 11.2$ & $85.7^{\mathrm{b}} \pm 14.2$ & $63.1 \pm 4.1$ \\
\hline AST $(\mathrm{U} / \mathrm{L})$ & $97.5 \pm 8.5$ & $129.1^{\mathrm{a}} \pm 24.6$ & $113.1 \pm 8.1$ \\
\hline ALT $(\mathrm{U} / \mathrm{L})$ & $37.2 \pm 5.2$ & $55.2^{\mathrm{a}} \pm 10.6$ & $42.2 \pm 8.8$ \\
\hline BUN $(\mathrm{mg} / \mathrm{dl})$ & $14.8 \pm 2.4$ & $25.6^{\mathrm{a}} \pm 3.3$ & $17.8 \pm 2.2$ \\
\hline $\begin{array}{l}\text { Creatinine } \\
(\mathrm{mg} / \mathrm{dl})\end{array}$ & $0.72 \pm 0.19$ & $1.1^{\mathrm{a}} \pm 0.11$ & $0.84 \pm 0.09$ \\
\hline
\end{tabular}

andicates statistical significance from the control group $(P<0.05)$; ${ }^{\mathrm{b}}$ indicates a significant difference from the control group $(P<0.01)$; ${ }^{\mathrm{c}}$ indicates statistical difference from control rats $(P<0.001) ;{ }^{\mathrm{d}}$ indicates statistical significance from the lead-intoxicated group $(P<0.05)$; and ${ }^{\mathrm{e}}$ indicates statistical significance from the lead-intoxicated group $(P<0.05)$.

of liver with well distinct liver sinusoids, normal portal triad, and the centering hepatocytes around the central vein (Figure 1A). Liver histopathological investigation of lead-intoxicated rats showed severe changes, such as disarrangement of liver sinusoids, with pyknotic nuclei, and early stages signs of hepatocellular necrosis (Figure 1B). The liver histopathological changes were less prominent in the lead-intoxicated rats treated with the CeNPs and intoxicated with lead acetate. In this group, there were necrotic nuclei and disarrangement of hepatic cords; however, these changes were lesser compared to the negative control group (Figure 1C). As shown in Figure 2, the periodic acid Schiff staining of the control rat's kidney was normal with intact glomeruli, bowmen capsule, and well-observed Bowman's space (Figure 2A). The leadacetate-treated rats showed a different histopathological pattern with diffuse hyaline casts in many parts of the nephron, such as distal and proximal tubules (Figure 3B). The lead-intoxicated rats, which received the CeNPs, had different histopathology and showed fewer signs of toxicity compared to the negative control rats (Figure 3C). The histological analysis of the testis was similar to the results of liver and kidney histopathology. As can be seen in Figure 3, treatment with CeNPs reduced leadinduced testicular histopathological changes in such a way that both lead-acetate treated groups showed signs of testicular toxicity (Figures $4 \mathrm{~B}$ and $4 \mathrm{C}$ ). In the current histopathological investigation, healthy control rats had normal testicular histology pattern with normal seminiferous tubules and healthy spermatogonium cells and the spermatic cords (Figure 4A). As shown in Figure $4 \mathrm{~B}$, the testis of rats treated with the lead acetate showed necrosis of germinal epithelium in the seminiferous tubules.

\section{Discussion}

Lead toxicity is a major health problem in developing countries. Many experiments have been focused on the toxic effects of lead and other heavy metals, including CeNPs. These studies showed undeniable evidence regarding the testicular toxicity of lead acetate in various animal models. CeNPs, as potential antioxidant agents, have been investigated in many previous studies for protecting against nephrotoxicity induced by heavy metals and reproductive toxicity (17). The results of the current experimental study were completely in agreement with previous studies conducted on hepatotoxicity-effects of lead acetate. Moreover, the current results showed that CeNPs - at proper dose- had no toxic effects on rats' biochemical parameters. The histopathological analysis confirmed the biochemical results. The results of $\mathrm{He}$ et al showed that CeNPs - at super nutritional levelshad no obvious toxic effects in rats (18). These results were in agreement with those of Hadrup et al (19). The difference in biological reports of CeNPs could be due to the difference in the dose, duration of study, and method of administrating CeNPs. In addition, CeNPs are easily absorbed from the skin, respiratory system, and gastrointestinal tract, and they could distribute to tissues quickly. However, they cannot easily penetrate

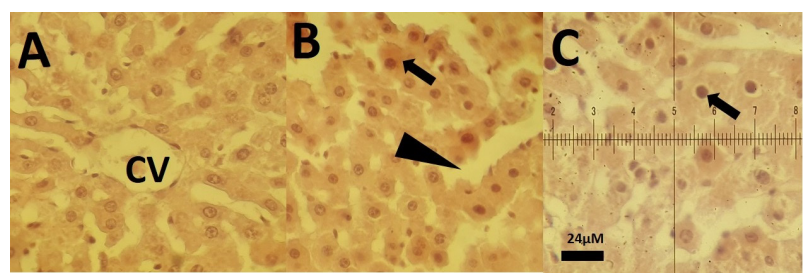

Figure 1. Light Micrograph of a Liver Section of Experimental Groups; A: Liver section of a normal control rat, B: liver section of a lead-treated rat; arrow shows disarrangement of hepatic cords, C: liver micrograph of a lead-intoxicated rat after treatment with CeNPs. (Periodic acid-Schiff staining microscopic magnification. $\times 40)$ bar $=24 \mu \mathrm{m}$.

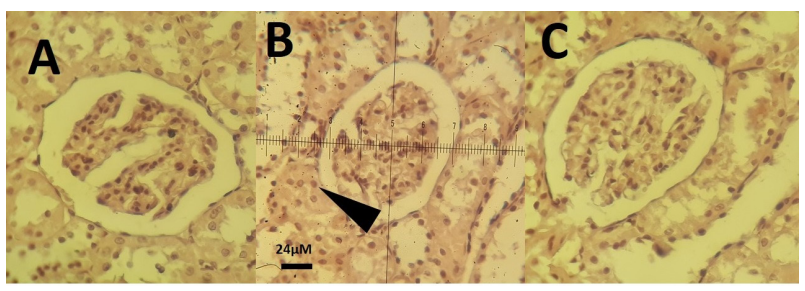

Figure 2. Periodic Acid-Schiff (PAS) Staining of Kidney Sections; A: kidney section of a normal control rat, B: lead-treated rat; arrow shows narrowing of proximal tubules, C: micrograph of a leadintoxicated rat after treatment with selenium nanoparticles. $(\mathrm{H} \& \mathrm{E}$ staining Microscopic magnification $\times 40$ ) bar $=24 \mu \mathrm{m}$.

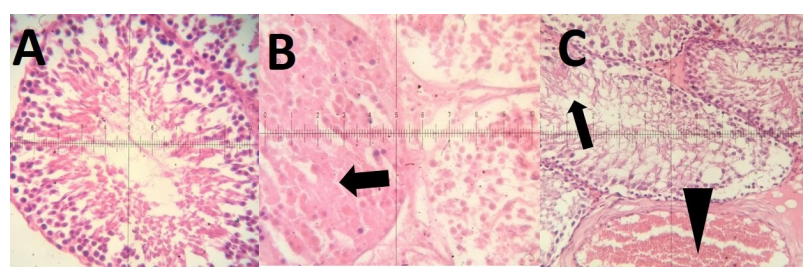

Figure 3. Light Micrographs of Testis Sections; A: control rats. B: lead-treated rat; arrow shows necrosis of seminiferous tubules. C: kidney section of a CeNPs-treated lead-intoxicated rat; arrow shows necrosis ( $\mathrm{H} \& \mathrm{E}$ staining. Microscopic magnification $\times 40)$. 


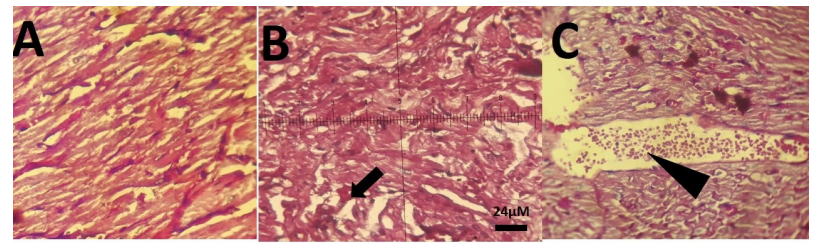

Figure 4. Light Micrographs of Heart Sections; A: control rats. B: lead-treated rat; arrow shows hypertrophy of heart muscles (Arrow). C: heart section of a CeNPs-treated lead-intoxicated rat; arrow shows congestion (arrow point) $(\mathrm{H} \& \mathrm{E}$ staining microscopic magnification $\times 40$ ).

specific organs such as the testes, thymus, and brain. Some studies have shown that in long-term periods, or when animals received high doses of NPs, a trace amount of them can cross the blood-testis barrier. The results of Hosseini et al showed the antioxidant and anti-apoptotic effects of CeNPs in rats' thyroid (20). Recent studies have also shown that CeNPs are less toxic than inorganic and organic Cerium oxide (21). In a previous study, the in vitro and in vivo toxicity assessment of CeNPs showed low cytotoxicity and good bactericidal activity (22). The dose-dependent toxicity of lead-acetate on various organs has been reported in earlier studies. In the current study, we observed significant histological alterations in different organs such as liver, testis, and kidney of animals. Earlier experiments have shown that CeNPs can induce histological changes in the liver and kidney of rats (23). Our histopathological investigation showed that lead could induce severe histological changes, a result which is in line with the previous studies (24). These changes were less pronounced in the CeNPs-treated lead-intoxicated rats. The effects of lead acetate and CeNPs on testis showed that these materials at specific doses could cross the bloodtestis barrier. The testis has a natural defense system called the blood-testis barrier, which protects germ cells from NPs and other exogenous materials in the circulatory or lymphatic systems. However, when rats received a specific dose of NPs, a trace amount of them penetrated through the blood-testis barrier to induce histological changes. Furthermore, recent studies have shown that CeNPs could induce cytotoxicity, inflammation, and blood barrier damage (25); thus, similar effects can be expected for the testis-blood barrier. Earlier experiments have shown that CeNPs can cross the blood vessels of the testis (26). In addition, some studies on NPs showed pro-oxidant effects of CeNPs in laboratory rodent models (27). The present study showed the efficacy of CeNPs in reducing lead acetate-intoxication side effects. Other studies have shown that selenium may reduce Arsenic or Cadmium toxicity by activating the nuclear factor erythroid 2-related factor 2 pathway. However, new experiments indicate that increased arsenic methylation could be the main mechanism by which selenium can protect the liver against arsenic toxicity (28). Selenium has shown strong hepatoprotective effects against hepatic oxidative damage induced by monocrotaline (29), acetaminophen
(30), diethylnitrosamine (31), and doxorubicin (32). CeNPs showed hepatoprotective and anti-inflammatory properties and reduced steatosis portal hypertension in rats with carbon tetrachloride-induced liver fibrosis (33). These rare metal NPs also showed interesting therapeutic effects such as antilipogenic effects in animal models of non-alcoholic fatty-liver disease (34). In the present study, lead exposure caused significant histopathological and biochemical changes in the heart. The main histological finding in the current study was cardiac hypertrophy, which is an indicator of cardiac toxicity. Previous studies have shown that chronic or acute lead exposure can cause endothelial injury, arteriosclerosis, infarction, and cardiomyopathy (35). The results of the present study showed that cerium oxide administration led to a decrease in lipid peroxidation, which could be considered as one of the mechanisms. Previous studies conducted on animal models showed that CeNPs could reduce the toxicity of several toxic metals such as cadmium and lead (36). The exact mechanism of action is not clearly understood, but it might be due to the antioxidant effects of this element. Another explanation is that these NPs could prevent the absorption of lead and other toxic metals from the digestive system; however, this hypothesis needs more scientific evaluations.

\section{Conclusion}

The CeNPs effectively reduced lead-inducing side effects on the liver, kidney, heart, and testis of rats. Further studies are required to determine the interaction of CeNPs with other heavy metals.

\section{Conflict of Interest Disclosures}

The authors declare that there is no conflict of interest

\section{Acknowledgments}

The authors would like to thank Mr. Salehi Moghdam for his excellent technical assistance.

\section{Ethical Statement}

All animal handling and injection procedures were performed following the ethical codes of the Animal Research Ethical Committee of the Faculty of Veterinary Medicine, University of Zabol (UOZ.ERC.1399).

\section{Authors' Contributions}

All authors equally participated in the proposal and design of the study, the acquisition of the data, data analysis, and interpretation.

\section{Funding/Support}

The current study received a grant from the University of Zabol (grant number: 9618-15).

\section{Informed Consent}

Not applicable.

\section{References}

1. Kumar A, Kumar A, M MSC, Chaturvedi AK, Shabnam AA, Subrahmanyam G, et al. Lead toxicity: health hazards, 
influence on food chain, and sustainable remediation approaches. Int J Environ Res Public Health. 2020;17(7):2179. doi: 10.3390/ijerph17072179.

2. Mitra P, Sharma S, Purohit P, Sharma P. Clinical and molecular aspects of lead toxicity: an update. Crit Rev Clin Lab Sci. 2017;54(7-8):506-28. doi: 10.1080/10408363.2017.1408562.

3. Kim JJ, Kim YS, Kumar V. Heavy metal toxicity: an update of chelating therapeutic strategies. J Trace Elem Med Biol. 2019;54:226-31. doi: 10.1016/j.jtemb.2019.05.003.

4. Hon KL, Fung CK, Leung AK. Childhood lead poisoning: an overview. Hong Kong Med J. 2017;23(6):616-21. doi: 10.12809/hkmj176214.

5. Li Y, Li P, Yu H, Bian Y. Recent advances (2010-2015) in studies of cerium oxide nanoparticles' health effects. Environ Toxicol Pharmacol. 2016;44:25-9. doi: 10.1016/j.etap.2016.04.004.

6. Farokhzad OC, Langer R. Impact of nanotechnology on drug delivery. ACS Nano. 2009;3(1):16-20. doi: 10.1021/ nn900002m.

7. Mohanraj VJ, Chen Y. Nanoparticles-a review. Trop J Pharm Res. 2006; 5(1):561-73. doi: 10.4314/tjpr.v5i1.14634.

8. Dhall A, Self W. Cerium oxide nanoparticles: a brief review of their synthesis methods and biomedical applications. Antioxidants (Basel). 2018;7(8):97. doi: 10.3390/ antiox7080097.

9. Maccarone R, Tisi A, Passacantando M, Ciancaglini M Ophthalmic applications of cerium oxide nanoparticles. J Ocul Pharmacol Ther. 2020;36(6):376-83. doi: 10.1089/ jop.2019.0105.

10. Das S, Dowding JM, Klump KE, McGinnis JF, Self W, Seal S. Cerium oxide nanoparticles: applications and prospects in nanomedicine. Nanomedicine (Lond). 2013;8(9):1483-508. doi: 10.2217/nnm.13.133.

11. Hirst SM, Karakoti AS, Tyler RD, Sriranganathan N, Seal S, Reilly CM. Anti-inflammatory properties of cerium oxide nanoparticles. Small. 2009;5(24):2848-56. doi: 10.1002/ smll.200901048.

12. Zhang R, Niu Y, Li Y, Zhao C, Song B, Li Y, et al. Acute toxicity study of the interaction between titanium dioxide nanoparticles and lead acetate in mice. Environ Toxicol Pharmacol. 2010;30(1):52-60. doi: 10.1016/j.etap.2010.03.015.

13. Wason MS, Zhao J. Cerium oxide nanoparticles: potential applications for cancer and other diseases. Am J Transl Res. 2013;5(2):126-31.

14. Hegazy MA, Maklad HM, Samy DM, Abdelmonsif DA, El Sabaa BM, Elnozahy FY. Cerium oxide nanoparticles could ameliorate behavioral and neurochemical impairments in 6-hydroxydopamine induced Parkinson's disease in rats. Neurochem Int. 2017;108:361-71. doi: 10.1016/j. neuint.2017.05.011.

15. Tu J, Zhu S, Li B, Xu G, Luo X, Jiang L, et al. Gegen Qinlian Decoction Coordinately Regulates PPAR $\gamma$ and PPARa to Improve Glucose and Lipid Homeostasis in Diabetic Rats and Insulin Resistance 3T3-L1 Adipocytes. Front Pharmacol. 2020;11:811. doi: 10.3389/fphar.2020.00811.

16. Ohkawa H, Ohishi N, Yagi K. Assay for lipid peroxides in animal tissues by thiobarbituric acid reaction. Anal Biochem. 1979;95(2):351-8. doi: 10.1016/0003-2697(79)90738-3.

17. Hirst SM, Karakoti A, Singh S, SelfW, Tyler R, Seal S, et al. Biodistribution and in vivo antioxidant effects of cerium oxide nanoparticles in mice. Environ Toxicol. 2013;28(2):107-18. doi: 10.1002/tox.20704.

18. He Y, Chen S, Liu Z, Cheng C, Li H, Wang M. Toxicity of selenium nanoparticles in male Sprague-Dawley rats at supranutritional and nonlethal levels. Life Sci. 2014;115(12):44-51. doi:10.1016/j.Ifs.2014.08.023.

19. Hadrup N, Loeschner K, Mandrup K, et al. Subacute oral toxicity investigation of selenium nanoparticles and selenite in rats. Drug Chem Toxicol. 2019;42(1):76-83. doi:10.1080/0 1480545.2018.1491589.

20. Hosseini A, Sharifi AM, Abdollahi M, Najafi R, Baeeri M, Rayegan $S$, et al. Cerium and yttrium oxide nanoparticles against lead-induced oxidative stress and apoptosis in rat hippocampus. Biol Trace Elem Res. 2015;164(1):80-9. doi: 10.1007/s12011-014-0197-z.

21. Kannan SK, Sundrarajan M. A green approach for the synthesis of a cerium oxide nanoparticle: characterization and antibacterial activity. Int J Nanosci. 2014;13(3):1450018. doi: 10.1142/s0219581x14500185.

22. Sharma JK, Srivastava P, Ameen S, Akhtar MS, Sengupta SK, Singh G. Phytoconstituents assisted green synthesis of cerium oxide nanoparticles for thermal decomposition and dye remediation. Mater Res Bull. 2017;91:98-107. doi: 10.1016/j. materresbull.2017.03.034.

23. Schwotzer D, Ernst H, Schaudien D, Kock H, Pohlmann G, Dasenbrock C, et al. Effects from a 90-day inhalation toxicity study with cerium oxide and barium sulfate nanoparticles in rats. Part Fibre Toxicol. 2017;14(1):23. doi: 10.1186/s12989017-0204-6.

24. El-Neweshy MS, El-Sayed YS. Influence of vitamin C supplementation on lead-induced histopathological alterations in male rats. Exp Toxicol Pathol. 2011;63(3):221-7. doi: 10.1016/j.etp.2009.12.003.

25. Ma JY, Zhao $H$, Mercer RR, Barger $M$, Rao M, Meighan $\mathrm{T}$, et al. Cerium oxide nanoparticle-induced pulmonary inflammation and alveolar macrophage functional change in rats. Nanotoxicology. 2011;5(3):312-25. doi: 10.3109/17435390.2010.519835.

26. Dkhil MA, Zrieq R, Al-Quraishy S, Abdel Moneim AE. Selenium nanoparticles attenuate oxidative stress and testicular damage in streptozotocin-induced diabetic rats. Molecules. 2016;21(11). doi: 10.3390/molecules21111517.

27. Aalapati S, Ganapathy S, Manapuram S, Anumolu G, Prakya BM. Toxicity and bio-accumulation of inhaled cerium oxide nanoparticles in CD1 mice. Nanotoxicology. 2014;8(7):78698. doi: 10.3109/17435390.2013.829877.

28. Zwolak I. The role of selenium in arsenic and cadmium toxicity: an updated review of scientific literature. Biol Trace Elem Res. 2020;193(1):44-63. doi: 10.1007/s12011-01901691-w.

29. Amin KA, Hassan MS, Awad el ST, Hashem KS. The protective effects of cerium oxide nanoparticles against hepatic oxidative damage induced by monocrotaline. Int J Nanomedicine. 2011;6:143-9. doi: 10.2147/ijn.s15308.

30. Córdoba-Jover B, Arce-Cerezo A, Ribera J, Pauta M, Oró D, Casals G, et al. Cerium oxide nanoparticles improve liver regeneration after acetaminophen-induced liver injury and partial hepatectomy in rats. J Nanobiotechnology. 2019;17(1):112. doi: 10.1186/s12951-019-0544-5.

31. Adebayo OA, Akinloye O, Adaramoye OA. Cerium oxide nanoparticles attenuate oxidative stress and inflammation in the liver of Diethylnitrosamine-treated mice. Biol Trace Elem Res. 2020;193(1):214-25. doi: 10.1007/s12011-019-016965.

32. Ibrahim HG, Attia N, Hashem F, El Heneidy MAR. Cerium oxide nanoparticles: in pursuit of liver protection against 
doxorubicin-induced injury in rats. Biomed Pharmacother. 2018;103:773-81. doi: 10.1016/j.biopha.2018.04.075.

33. Oró D, Yudina T, Fernández-Varo G, Casals E, Reichenbach V, Casals $\mathrm{G}$, et al. Cerium oxide nanoparticles reduce steatosis, portal hypertension and display anti-inflammatory properties in rats with liver fibrosis. J Hepatol. 2016;64(3):691-8. doi: 10.1016/j.jhep.2015.10.020.

34. Carvajal S, Perramón M, Oró D, Casals E, Fernández-Varo G, Casals G, et al. Cerium oxide nanoparticles display antilipogenic effect in rats with non-alcoholic fatty liver disease. Sci Rep. 2019;9(1):12848. doi: 10.1038/s41598-
019-49262-2.

35. Vaziri ND. Mechanisms of lead-induced hypertension and cardiovascular disease. Am J Physiol Heart Circ Physiol. 2008;295(2):H454-65. doi: 10.1152/ajpheart.00158.2008.

36. Al-Kinani A, Eftekhari M, Gheibi M, Chamsaz M. Polyanilinecoated cerium oxide nanoparticles as an efficient adsorbent for preconcentration of ultra-trace levels of cadmium (II) followed by electrothermal atomic absorption spectrometry. Spectrosc Lett. 2018;51(6):287-96. doi: 10.1080/00387010.2018.1473884. 